Article

\title{
Carbon Quantum Dots Encapsulated Molecularly Imprinted Fluorescence Quenching Particles for Sensitive Detection of Zearalenone in Corn Sample
}

\author{
Manyu Shao ${ }^{1,+}$, Ming Yao ${ }^{1,+}$, Sarah De Saeger ${ }^{2} \mathbb{}$, Liping Yan ${ }^{1, *}$ and Suquan Song ${ }^{1, *}$ \\ 1 College of Veterinary Medicine, Nanjing Agricultural University, Nanjing 210095, China; \\ shaomanyu@outlook.com (M.S.); 2017807151@njau.edu.cn (M.Y.) \\ 2 Centre of Excellence in Mycotoxicology and Public Health, Ghent University, Ottergemsesteenweg 460, \\ 9000 Ghent, Belgium; sarah.desaeger@ugent.be \\ * Correspondence: yanliping@njau.edu.cn (L.Y.); suquan.song@njau.edu.cn (S.S.); \\ Tel.: +86-25-8439-5789 (L.Y.); +86-25-8439-5789 (S.S.) \\ $\dagger$ These authors contributed equally to this work.
}

Received: 1 October 2018; Accepted: 26 October 2018; Published: 28 October 2018

\begin{abstract}
An eco-friendly and efficient one-step approach for the synthesis of carbon quantum dots (CDs) that encapsulated molecularly imprinted fluorescence quenching particles (MIFQP) and their application for the determination of zearalenone (ZEA) in a cereal sample are described in this study. CDs with high luminescence were first synthesized, and then encapsulated in the silica-based matrix through a non-hydrolytic sol-gel process. The resulting ZEA-imprinted particles exhibited not only an excellent specific molecular recognition of ZEA, but also good photostability and obvious template binding-induced fluorescence quenching. Under the optimized conditions, the fluorescence intensity of MIFQP was inversely proportional to the concentration of ZEA. By validation, the detection range of these fluorescence quenching materials for ZEA was between 0.02 and $1.0 \mathrm{mg} \mathrm{L}^{-1}$, and the detection limit was $0.02 \mathrm{mg} \mathrm{L}^{-1}(\mathrm{~S} / \mathrm{N}=3)$. Finally, the MIFQP sensor was successfully applied for ZEA determination in corn with recoveries from $78 \%$ to $105 \%$ and the relative standard deviation (RSD \%) was lower than $20 \%$, which suggests its potential in actual applications.
\end{abstract}

Keywords: molecularly imprinted fluorescence quenching particles; carbon quantum dots; non-hydrolytic sol-gel; zearalenone; fluorescence quenching

Key Contribution: New eco-friendly, molecularly imprinted fluorescence quenching particles for zearalenone were developed and characterized, which has been proven to be promising for ZEA quantification in a corn sample.

\section{Introduction}

Zearalenone (ZEA) contaminates cereals and derived products across the world [1]. Toxic effects of ZEA include infertility, teratogenesis, neurotoxicity, carcinogenicity, and abortion [2,3]. Therefore, the International Agency for Research on Cancer (IARC) has listed ZEA as a group III carcinogen [4], and the increasing awareness of mycotoxins has increased the need for the accurate and reliable analysis of ZEA in cereal products.

Over the past few years, various methods, including thin-layer chromatography (TLC) [5], the enzyme-linked immunosorbent assay (ELISA) [6], and high-performance liquid chromatography (HPLC) based instrumental methods have been developed for ZEA detection [7-9]; however, these methods have some unavoidable limitations. For example, TLC lacks selectivity or sensitivity in complex matrices [10]; chromatographic-based methods need expensive instruments and professional 
operators [11]; and immunochemical techniques, such as ELISA, need highly specific antibodies, which are difficult to produce and conserve.

Molecularly imprinted polymers (MIPs) are synthetic highly cross-linked polymers, which form the specific cavities that are complementary to the size and shape of the template molecules during the copolymerization [12-17]. The resulting polymer can specifically rebind the template molecules in suitable solvents $[18,19]$. So far, there are two major preparation strategies. One is the silica-based system and the other is the organic-polymer-based system. The organic-polymer-based system has been widely used due to the availability of different monomers and its excellent stability in different $\mathrm{pH}$ environments. However, the polymers prepared using this method may shrink or swell in different solvents, which certainly affects their recognition of the template [20]. Silica-based monoliths may show good solvent resistance. However, these materials are often synthesized via hydrolytic sol-gel technology [21,22], which may crack or shrink during drying. Therefore, a feasible strategy to produce silica-based MIPs using the non-hydrolytic sol-gel (NHSG) technique has been developed, which can not only overcome the above mentioned shortcomings of the MIPs, but also decrease non-selective absorbance [23].

Recently, fluorescence-based sensing has received great attention for its application in biosensors owing to its low cost, excellent sensitivity, and fast response [24]. Among these sensory materials, quantum dots (QDs) have been widely used [25-27]. However, due to the usage of poisonous heavy metals in their synthesis, QDs can threaten human health and pollute the environment. Therefore, an alternative to QDs, new luminescent materials, such as graphene quantum dots (GQDs) and carbon quantum dots (CDs), have received more attention due to their high water solubility, low toxicity, easy functionalization, and excellent biocompatibility [28-32]. Because CDs can be prepared by the pyrolysis of organic molecules subjected to high temperatures, while the precursors of GQDs are mainly graphene-based materials, CDs is more popular in fluorescence-based sensing [33]. However, CDs are rarely applied in the food safety testing field [34,35]. The main obstacle lies in the immobilization of CDs in the polymers, which allows the permeation of analyte while avoiding the leaching of CDs. Therefore, silica-based MIPs may be a promising matrix that could efficiently protect the photoluminescent (PL) properties of CDs, without affecting the binding of the target molecules. However, in order to intensify the interaction between the silica matrix and CDs, the CDs should be previously pretreated with organosilane, which means that the preparation process of such polymers requires at least two steps.

In this study, a novel eco-friendly fluorescence quenching material has been developed as a ZEA sensor through an efficient one-step NHSG molecular imprinting process. The synthesis of MIFQP is demonstrated in Figure 1. This polymer integrates the accurate selectivity of MIPs with the high fluorescence (FL) stability of CDs. In the sensor, CDs work as antennas for sample recognition and signal amplification, and polymers provide specific binding sites for ZEA. When ZEA is present in the sample, the FL of CDs is blocked. The degree of FL quenching is proportional to ZEA concentration. Importantly, with these materials, the simple, sensitive, and fast detection of ZEA in a corn sample can be realized. 


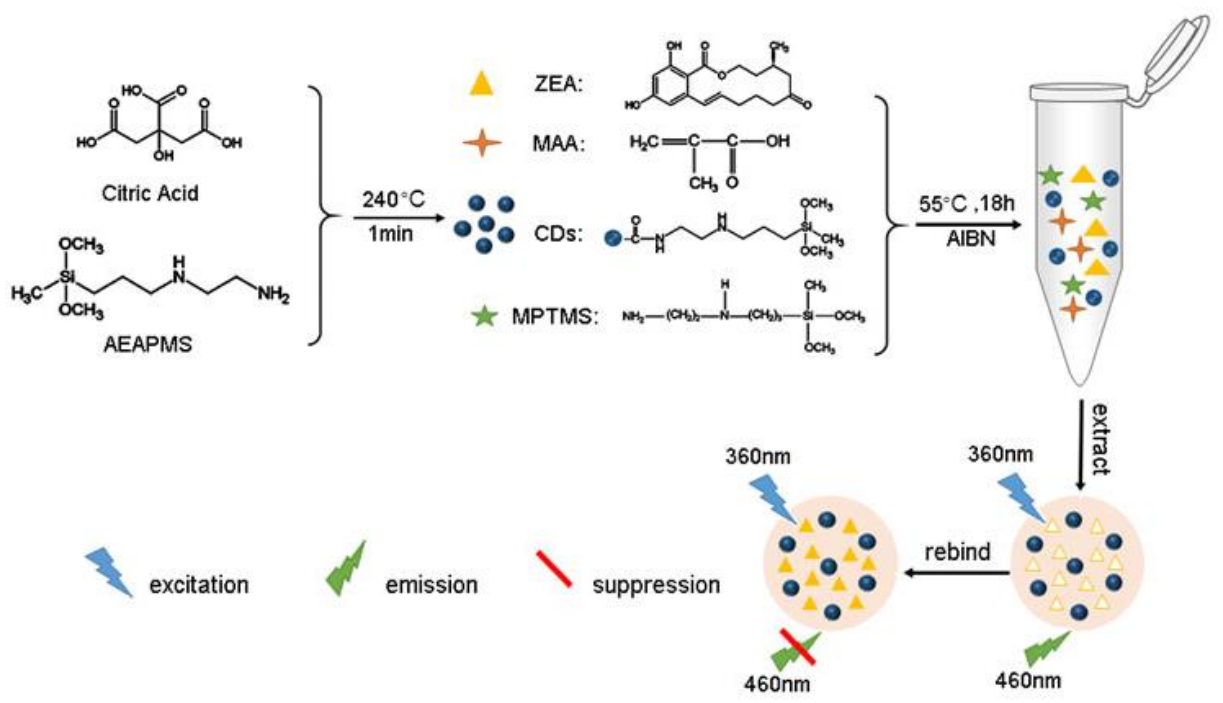

Figure 1. Preparation of MIFQP.

\section{Results and Discussion}

\subsection{Preparation and Characterization of $C D s$}

Multi-aminosilane (AEAPMS) modified CDs with a highly fluorescent property were prepared by an efficient one-step reaction and characterized by FT-IR, DLS, and fluorescence spectroscopy, respectively. As demonstrated in Figure 2A, CD microspheres with a well-distributed size were obtained, with an average particle size of $3.4 \mathrm{~nm}$. As reported, during CD synthesis, a surface passivation reaction occurred between the amine groups of AEAPMS and the carboxyl groups derived from the pyrolysis of anhydrous citric acid [36]. Therefore, the acylation was confirmed by FT-IR spectroscopy (Figure 2B). Different from AEAPMS, the spectrum of CDs exhibited two characteristic peaks at $1650 \mathrm{~cm}^{-1}$ and $1565 \mathrm{~cm}^{-1}$, which were similar to the vibrations of amide I and II, respectively. These typical signals of $C=O N R$ vibrations indicated the successful passivation reaction on the $C D$ surface. In addition, the CDs showed an evident adsorption peak at $360 \mathrm{~nm}$ (Figure 2C). When excited in the spectrum of 340-400 nm, the CDs showed strong blue PL at $460 \mathrm{~nm}$, which was strongly dependent on the excitation energy.
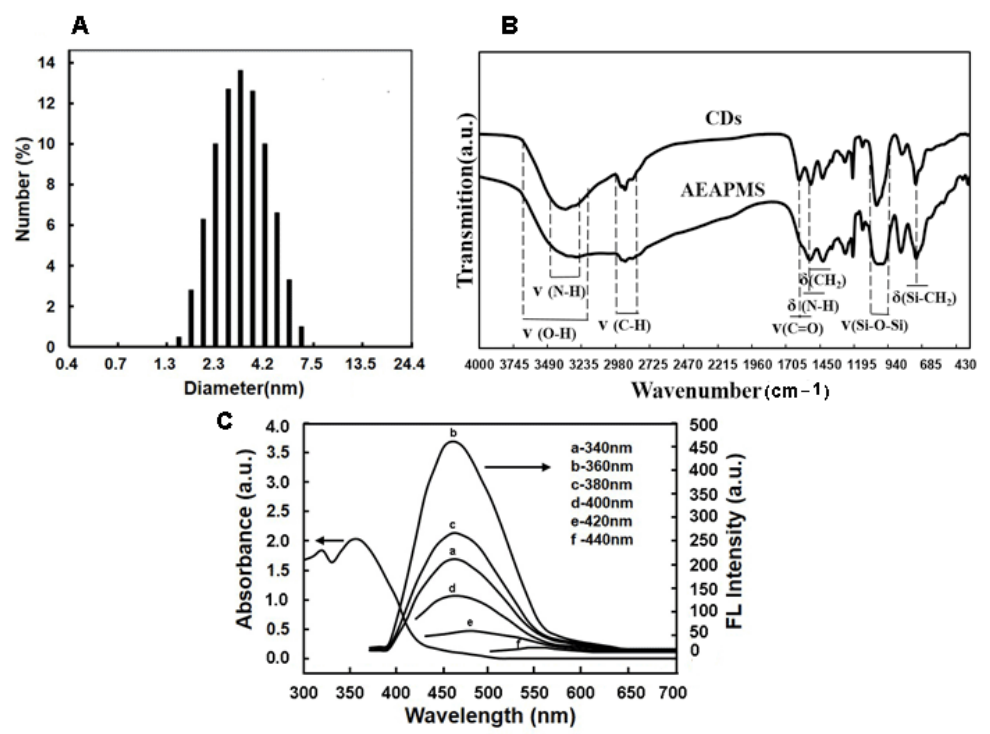

Figure 2. (A) Size distribution of CDs (obtained from the DLS measurement in ethanol); (B) FT-IR spectra of AEAPMS and CDs; (C) Absorption and PL emission spectra of the CDs in ethanol. 


\subsection{MIFQP Preparation and Characterization}

A ZEA imprinted fluorescence quenching mesoporous structured polymer encapsulated with CDs was prepared by the NHSG route in this study, where ZEA, methacrylic acid (MAA), azobisisobutyronitrile (AIBN), and $\gamma$-methacryloxypropyltrimethoxysilane (MPTMS) acted as the template molecule, functional monomer, free radical initiator, and cross-linker, respectively. Briefly, MAA catalyzed the condensation reaction between MPTMS and the passivated CDs, and then an Si-O-Si framework was formed. Simultaneously, with the thermal initiation of AIBN, carbon-carbon double bonds between MAA and MPTMS polymerized. Consequently, CDs with high luminescence were encapsulated in the silica-based materials. After eluting the template molecule by acetone, the MIFQP with specific recognition sites for ZEA was generated. As shown in Figure 3A,B, both MIFQP and a control polymer exhibited a similar rough surface, with a narrowly distributed particle size around $100 \mu \mathrm{m}$. FT-IR spectra of the imprinted and control polymer further confirmed that ZEA was successfully embedded into the MIFQP (Figure 3C). In detail, the strong broad peaks at $3371 \mathrm{~cm}^{-1}$ and $1651 \mathrm{~cm}^{-1}$ resulted from the stretching vibration of hydroxyl and carbonyl groups of the ZEA molecule (Figure 3C-a). These peaks were also obvious for MIFQP before extraction (Figure 3C-b), but disappeared after the extraction of ZEA (Figure 3C-c). Therefore, after polymerization, ZEA was successfully grafted into the silica matrix, and could be washed out with a suitable solvent. In addition, the strong peak around $1067 \mathrm{~cm}^{-1}$ was ascribed to the Si-O-Si asymmetric stretching, and Si-O vibrations showed characteristic peaks at $773 \mathrm{~cm}^{-1}$ and $453 \mathrm{~cm}^{-1}$. All of these characteristics further confirmed that through NHSG condensation of the silane reagents, CDs were successfully encapsulated into the imprinted polymer. Besides, similar FT-IR spectra between extracted MIFQP and control polymers indicated that they have a similar composition, and the template molecules had been removed with extraction (Figure 3C-c,d). Furthermore, in order to confirm the successful encapsulation of CDs into the MIP, the fluorescence excitation and emission spectra of MIFQP were tested. The results showed that, consistent with the results demonstrated in Figure 2C, when excited at $360 \mathrm{~nm}$, the synthesized MIFQP showed an obvious fluorescence emission peak at $460 \mathrm{~nm}$ (Figure 3D), suggesting that the fluorescence properties of CDs were not influenced during the preparation process due to the optically transparent and inert property of silica [37].
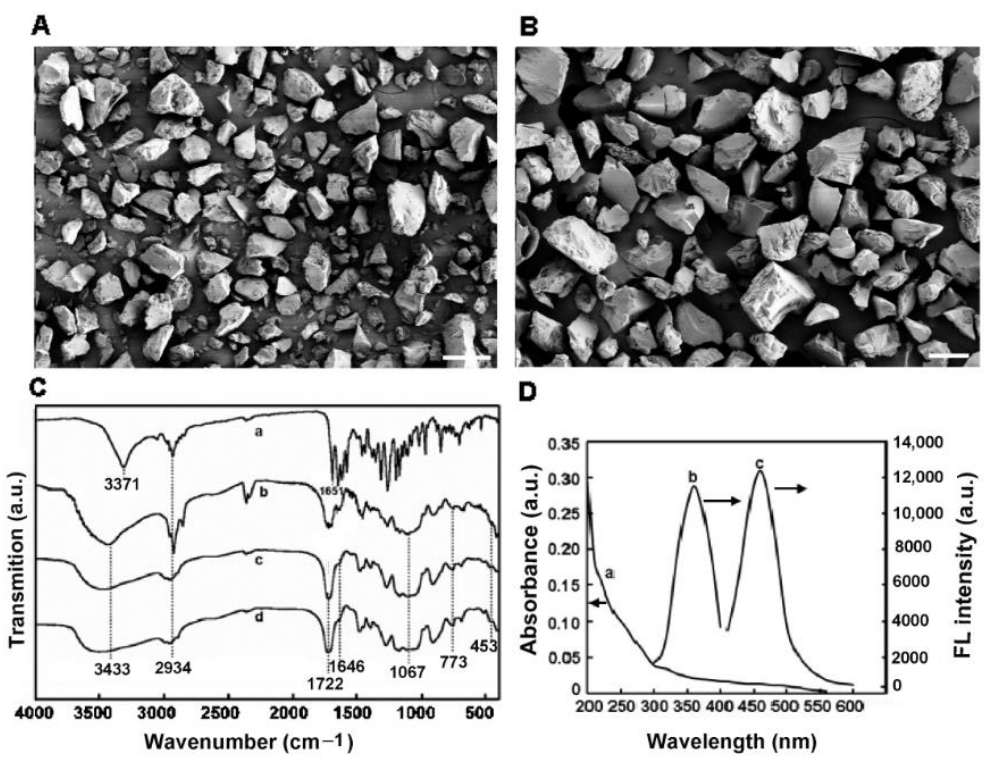

Figure 3. SEM images of (A) MIFQP and (B) control; (C) FT-IR spectra of (a) ZEA, MIFQP (b) before and (c) after extraction, and (d) control polymer after extraction; (D) (a) UV-vis absorption, (b) FL excitation, and (c) emission spectra of MIFQP. Scale bar $=100 \mu \mathrm{m}$. 
The synthesized MIFQP was then evaluated for ZEA sensing. As shown in Figure 4A, after removal of the template (Figure 4A-c), the fluorescence intensity of MIFQP almost reached the same level as the control (non-imprinted polymers) (Figure 4A-a). After the addition of ZEA, the fluorescence of MIFQP was sharply quenched (Figure $4 \mathrm{~A}-\mathrm{d}$ ), and the quenching was inversely proportional to the amount of ZEA (Figure 4A). The mechanism of FL quenching may be ascribed to the electron transfer from the acceptor to donor molecules in solution [38,39] in which ZEA and CDs may act as the electron acceptor and donor, respectively. The MIFQP can bind ZEA, and the bound ZEA will accept electrons from CDs in the MIPs; therefore, the FL of MIFQP could be effectively quenched after the incorporation with ZEA molecules. Furthermore, the FL photostability of the polymers was investigated in the study. The result indicated that the fluorescence intensities of MIFQP suspension were stable within 10 consecutive days (standard deviation (RSD) of $1.19 \%$ ) when the concentration of ZEA was $312.5 \mu \mathrm{g} \mathrm{mL}^{-1}$ (Figure $4 \mathrm{~B}$ ), which indicates the excellent photostability of the obtained MIFQP.
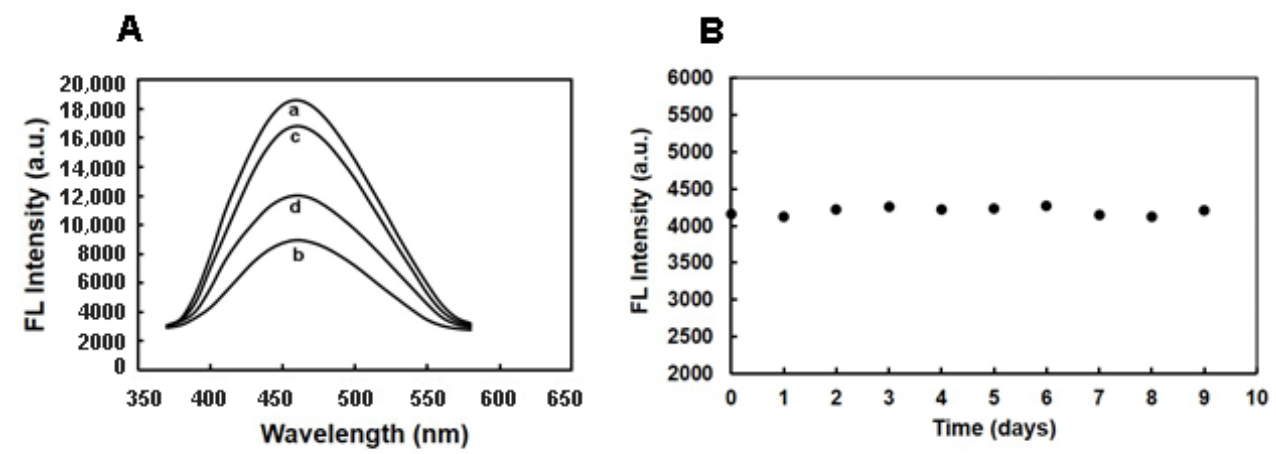

Figure 4. (A) FL spectra of (a) control polymer, MIFQP (b) before and (c) after removal of template, and (d) MIFQP with addition of ZEA (500 $\mathrm{g} \mathrm{mL}^{-1}$ of ZEA in water); (B) Stability of fluorescence emission property of MIFQP in water (the concentration of ZEA is $312.5 \mu \mathrm{g} \mathrm{mL}^{-1}$ ).

\subsection{Selectivity and Sensitivity of MIFQP Experiments}

The selectivity and sensitivity of MIFQP were evaluated by measuring their fluorescent responses to ZEA and other mycotoxins such as AFB1, DON, OTA, T-2, patulin, and BEA $\left(250 \mu \mathrm{g} \mathrm{mL}^{-1}\right)$. As indicated in Figure 5, ZEA could obviously quench the FL of the imprinted polymer $\left(F_{0} / F-1=0.834\right)$, which was 3.97 times higher than that of the control (non-imprinted polymers). However, for the other compounds, a similar fluorescence change was observed for MIFQP and the control, suggesting that the MIFQP are ZEA specific. Additionally, the fluorescence quenching efficiency of ZEA towards the materials was much higher than other mycotoxins, which indicates the high selectivity of the imprinted polymers. The specificity of MIFQP towards ZEA may be attributed to the shapes of the cavities and presence of imprinted binding sites, which were formed during the polymerization. However, the fluorescence quenching of other compounds was mainly because of the non-specific bindings.

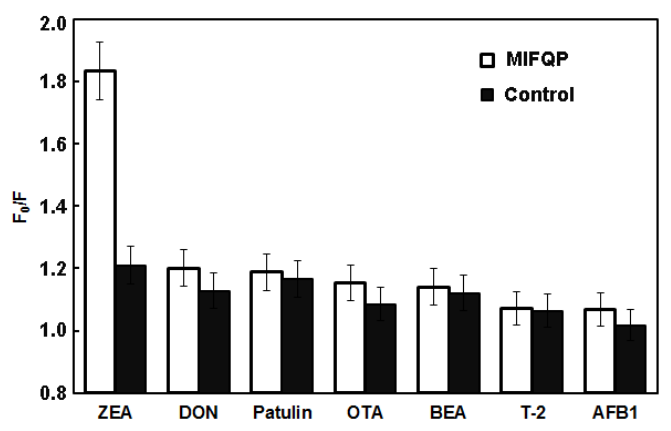

Figure 5. Selectivity of MIFQP and control for ZEA, DON, patulin, OTA, BEA, T-2, and AFB1. The concentration was $250 \mu \mathrm{g} \mathrm{mL}^{-1}$ of each compound. 


\subsection{Validation and Application of MIFQP}

In order to evaluate the applicability of the proposed polymer for ZEA determination in real samples, we added ZEA into the raw corn samples before sample preparation, and then analyzed the spiked samples. As indicated in the figure, with increasing ZEA concentrations, the fluorescence intensity gradually decreases for MIFQP. The fluorescence quenching in this system was in accordance with the Stern-Volmer equation [40], $F_{0} / F=1+K_{\text {Sv }} C_{\text {ZEA }}$, where $F_{0}$ and $F$ represent the fluorescence intensities without or with the template (quencher), respectively; $K_{\mathrm{sv}}$ is the quenching constant of the quencher; and $C_{\text {ZEA }}$ is the concentration of quencher. As demonstrated in Figure 6A, the MIFQP exhibited an obviously higher fluorescence quenching efficiency. As shown in Figure 6B, the signal $\left(F_{0} / F-1\right)$ of the assay exhibited a linear correlation to ZEA concentration. In detail, the linear plots of fluorescence quenching of MIFQP versus ZEA concentrations were depicted within the concentration range of $0.02-1.0 \mathrm{mg} \mathrm{L}^{-1}$, with a correlation coefficient of 0.9923 . The LOD of ZEA for MIFQP was $20 \mu \mathrm{g} \mathrm{L}^{-1}$ and the LOQ was $60 \mu \mathrm{g} \mathrm{L}{ }^{-1}(S / N=3)$, which is higher than the ELISA or HPLC-MS/MS, but is far below the maximum limits (MLs) $\left(200 \mu \mathrm{g} \mathrm{kg}^{-1}\right)$ of ZEA in a cereal sample set by the Europe Union [41]. At three spiking concentrations $\left(200,400\right.$, and $\left.800 \mu \mathrm{g} \mathrm{kg}^{-1}\right)$, the recoveries ranged from $78 \%$ to $105 \%$, with the RSD lower than $20 \%$ (Table 1 ).

Once the method was validated, MIFQP sensors were applied to investigate the occurrence of ZEA in 22 corn samples. The results showed that $77 \%(17 / 22)$ of samples were found to be positive for ZEA. Among these ZEA contaminated samples, four were contaminated above the MLs. Therefore, the ZEA specific MIFQP biosensor developed in the study is promising for actual applications.
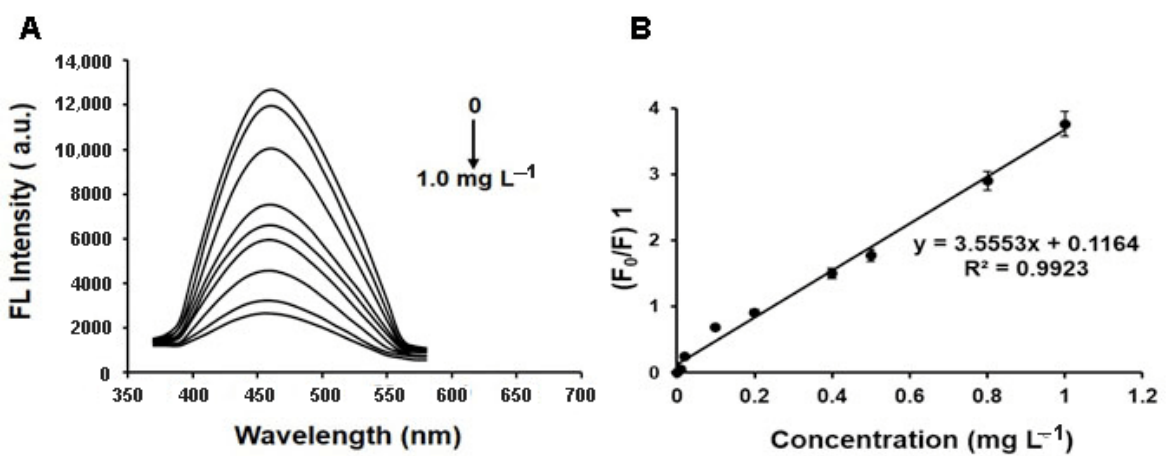

Figure 6. (A) MIFQP upon addition of the indicated concentration of ZEA; (B) Calibration curves of MIFQP for ZEA.

Table 1. Overview of the recoveries, repeatability $\left(\mathrm{RSD}_{\mathrm{r}}\right)$, reproducibility $\left(\mathrm{RSD}_{\mathrm{R}}\right)$, and limits of detection and quantitation (LOD and LOQ) of ZEA in corn determined with MIFQP. $(n=3)$.

\begin{tabular}{|c|c|c|c|c|c|c|}
\hline Sample & $\begin{array}{c}\text { Concentration of ZEA } \\
\left(\mathrm{mg} \mathrm{L} \mathrm{L}^{-1}\right)\end{array}$ & $\begin{array}{l}\text { Recovery } \\
(\%)\end{array}$ & $\mathrm{RSD}_{\mathrm{r}}$ & $\mathrm{RSD}_{\mathrm{R}}$ & $\underset{\left(\mathrm{mg} \mathrm{L}^{-1}\right)}{\text { LOD }}$ & $\begin{array}{c}\mathrm{LOQ} \\
\left(\mathrm{mg} \mathrm{L}^{-1}\right)\end{array}$ \\
\hline 1 & 0.2 & 105.1 & 13.3 & 16.3 & \multirow{3}{*}{0.02} & \multirow{3}{*}{0.06} \\
\hline 2 & 0.4 & 78.2 & 10.1 & 14.1 & & \\
\hline 3 & 0.8 & 90.7 & 8.7 & 12.9 & & \\
\hline
\end{tabular}

\section{Conclusions}

In summary, a one-step method for the preparation of hydrophilic, highly sensitive, and selective fluorescence quenching materials was developed in this study. The FL sensor showed high specificity and excellent optical readout. The MIFQP was confirmed to be applicable for ZEA determination in corn samples. In considering the simple synthesis of particles and their excellent dispersion and fluorescence properties in aqueous solution, we believe that such polymers may be promising in the analysis of other contaminants. 


\section{Materials and Methods}

\subsection{Materials}

$\mathrm{N}$-( $\beta$-aminoethyl)- $\gamma$-aminopropylmethyldimethoxysilane (AEAPMS, 97\%), azobisisobutyronitrile (AIBN, 99\%), methacrylic acid (MAA, 99\%), $\gamma$-methacryloxypropyltrimethoxysilane (MPTMS, 99\%), and anhydrous citric acid (99\%) were purchased from Sigma-Aldrich (St. Louis, MO, USA, https: //www.sigmaaldrich.com/). Acetonitrile and methanol were obtained from Merck (Darmstadt, Germany, http:/ / www.merckmillipore.com/). Zearalenone (ZEA, 99\%), deoxynivalenol (DON, 99\%), ochratoxin (OTA, 99\%), aflatoxin B1 (AFB1, 99\%), patulin (99\%), beauvericin (BEA, 99\%), and T-2 (99\%) were from Fermentek (Jerusalem, Israel, https:/ / www.fermentek.com/). Acetic acid (99\%) and other chemical reagents were provided by Sinopharm Co. (Shanghai, China, http:/ / www.sinopharmholding. $\mathrm{com} / \mathrm{en} /$ ). All reagents were of analytical grade.

\subsection{Instruments and Measurements}

FL measurements were performed with an Infinite M200 PRO instrument (TECAN, Switzerland, https://www.tecan.com/), while UV-vis spectra analyses were performed on a NanoDrop 2000 Spectrophotometer (https: / www.thermofisher.com). Fourier transform infrared (FT-IR) spectroscopic tests were performed on a Bio-Rad FTS6000 spectrophotometer (www.bio-rad.com/). Scanning electron microscopy (SEM), Hitachi SU1510, was used to characterize the surface morphologies of MIPs and NIPs (https:/ / www.hitachi-hightech.com). A dynamic laser scattering (DLS) spectrometer (Zetasizer Nano ZS90, https://www.malvernpanalytical.com) was used to determine the size distribution of the MIFQP.

\subsection{CDs and MIFQP Synthesis}

CDs were synthesized according to Wang et al. [34]. Briefly, AEAPMS (10 mL) was put into a 100-mL three-necked flask and degassed for 5 min with nitrogen. Then, the flask was heated to $240^{\circ} \mathrm{C}$, and $0.5 \mathrm{~g}$ citric acid anhydrous was quickly added, followed by vigorous stirring. After cooling to room temperature, the synthesized materials were purified three times with petroleum ether. Afterwards, the polymer was produced via an NHSG process. A total of $0.05 \mathrm{mmol}$ ZEA, $100 \mu \mathrm{L} \mathrm{CDs}$, and $2.0 \mathrm{mmol}$ MAA were sequentially dissolved in a solution of $4.0 \mathrm{~mL}$ chloroform containing $1.0 \mathrm{~mL}$ acetonitrile. The mixture was sealed and stirred in the dark for $1 \mathrm{~h}$. Then, $3.0 \mathrm{mmol}$ MPTMS and $20.0 \mathrm{mg}$ AIBN were added. Before being sealed, the mixture was sonicated for $10 \mathrm{~min}$ and purged with oxygen-free nitrogen for $5 \mathrm{~min}$. Finally, the polymerization occurred at $55^{\circ} \mathrm{C}$ for $18 \mathrm{~h}$ in the dark. The resulting bulk polymers were grounded mechanically and wet-sieved with acetone through a 200-mesh steel sieve. After extraction with acetone/acetic acid $(8: 2, v / v)$ for $48 \mathrm{~h}$ with a soxhlet apparatus (removal of ZEA), the MIFQP was dried at $60{ }^{\circ} \mathrm{C}$ for $12 \mathrm{~h}$. Control polymers were simultaneously prepared as described above without the addition of a ZEA template.

\subsection{FL Measurements}

In order to measure the recognition capacity of polymers and the control, $50 \mu \mathrm{g}$ of each compound was separately dispersed in $150 \mu \mathrm{L}$ acetic acid (5\%) with different concentrations of ZEA in a Corning Costar 96-Well Black Polystyrene Plate, and shaken for $4 \mathrm{~h}$ at room temperature. The adsorption of MIFQP and control was measured by fluorescence spectroscopy with excitation at $360 \mathrm{~nm}$. The excitation and emission slits were $5 \mathrm{~nm}$.

\subsection{Sample Pretreatment}

Maize samples were purchased from local markets in Shanghai, China. For sample preparation, $2 \mathrm{~g}$ of finely ground corn and $5 \mathrm{~mL}$ water were put into a $50 \mathrm{~mL}$ polypropylene centrifuge tube. Then, the tube was vortexed for $1 \mathrm{~min}$, followed by the addition of $5 \mathrm{~mL}$ of acidified ACN (1\% FA). 
To completely disperse the sample, the tube was vigorously shaken for $30 \mathrm{~min}$, and then anhydrous $\mathrm{MgSO}_{4}(1 \mathrm{~g})$ and $\mathrm{NaCl}(0.25 \mathrm{~g})$ were added, followed by immediate shaking for $30 \mathrm{~s}$ and centrifuging for $5 \mathrm{~min}$ at $6000 \times \mathrm{g}$. The supernatant was transferred into another $5 \mathrm{~mL}$ tube and evaporated at $40{ }^{\circ} \mathrm{C}$ with nitrogen. The residue was then resolved with $1 \mathrm{~mL}$ methanol and stored at $4{ }^{\circ} \mathrm{C}$ until analysis.

\subsection{Method Validation and Quality Assurance}

A spiked corn sample was used to validate the method. The evaluated parameters included linearity, recovery, repeatability $\left(\mathrm{RSD}_{\mathrm{r}}\right)$, reproducibility $\left(\mathrm{RSD}_{\mathrm{R}}\right)$, limits of detection (LODs), and limits of quantification (LOQs).

Author Contributions: Conceptualization, S.S.; Methodology, M.S. and M.Y.; Validation, M.Y. and L.Y.; Investigation, M.S. and L.Y.; Writing—original draft preparation, M.S.; Writing—review and editing, S.S. and S.D.S.; Project administration, S.S.; Funding acquisition, S.S.

Funding: This research was supported by the National Key R\&D Program, grant number 2016YFD0501200; the Natural Science Foundation of Jiangsu Province, grant number 2BK20161452; the Key Program of Science and Technology Planning of Guangdong Province, grant number 22017B020202010; and NAU International Cooperation and Cultivation Project, grant number 2018-AF-20.

Conflicts of Interest: The authors declare no conflict of interest.

\section{References}

1. Zhan, S.; Huang, X.; Chen, R.; Li, J.; Xiong, Y. Novel fluorescent ELISA for the sensitive detection of zearalenone based on $\mathrm{H}_{2} \mathrm{O}_{2}$-sensitive quantum dots for signal transduction. Talanta 2016, 158, 51-56. [CrossRef] [PubMed]

2. Ahamed, S.; Foster, J.S.; Bukovsky, A.; Wimalasena, J. Signal transduction through the Ras/Erk pathway is essential for the mycoestrogen zearalenone-induced cell-cycle progression in MCF-7 cells. Mol. Carcinog. 2001, 30, 88-98. [CrossRef]

3. Zinedine, A.; Soriano, J.M.; Moltó, J.C.; Mañes, J. Review on the toxicity, occurrence, metabolism, detoxification, regulations and intake of zearalenone: An oestrogenic mycotoxin. Food Chem. Toxicol. 2007, 45, 1-18. [CrossRef] [PubMed]

4. Shier, W.T.; Xie, W.M.C.; Shier, A.C. Structure-activity relationships for human estrogenic activity in zearalenone mycotoxins. Toxicon 2001, 39, 1435-1438. [CrossRef]

5. Hadiani, M.R.; Yazdanpanah, H.; Ghazikhansari, M.; Cheraghali, A.M.; Goodarzi, M. Survey of the natural occurrence of zearalenone in maize from northern Iran by thin-layer chromatography densitometry. Food Addit. Contam. 2003, 20, 380-385. [CrossRef] [PubMed]

6. Pei, S.C.; Lee, W.J.; Zhang, G.P.; Hu, X.F.; Eremin, S.A.; Zhang, L.J. Development of anti-zearalenone monoclonal antibody and detection of zearalenone in corn products from China by ELISA. Food Control 2013, 31, 65-70. [CrossRef]

7. Ok, H.E.; Choi, S.W.; Kim, M.; Chun, H.S. HPLC and UPLC methods for the determination of zearalenone in noodles, cereal snacks and infant formula. Food Chem. 2014, 163, 252-257. [CrossRef] [PubMed]

8. Songsermsakul, P.; Sontag, G.; Cichnamarkl, M.; Zentek, J.; Razzazifazeli, E. Determination of zearalenone and its metabolites in urine, plasma and faeces of horses by HPLC-APCI-MS. J. Chromatogr. B 2006, 843, 252-261. [CrossRef] [PubMed]

9. Wang, Y.; Xiao, C.; Guo, J.; Yuan, Y.; Wang, J.; Liu, L.; Yue, T. Development and application of a method for the analysis of 9 mycotoxins in maize by HPLC-MS/MS. J. Food Sci. 2013, 78, 1752-1756. [CrossRef] [PubMed]

10. Turner, N.W.; Subrahmanyam, S.; Piletsky, S.A. Analytical methods for determination of mycotoxins: A review. Anal. Chim. Acta 2009, 632, 168-180. [CrossRef] [PubMed]

11. Mao, Y.; Bao, Y.; Han, D.; Li, F.; Niu, L. Efficient one-pot synthesis of molecularly imprinted silica nanospheres embedded carbon dots for fluorescent dopamine optosensing. Biosens. Bioelectron. 2012, 38, 55-60. [CrossRef] [PubMed]

12. Wei, J.R.; Ni, Y.L.; Zhang, W.; Zhang, Z.Q.; Zhang, J. Detection of glycoprotein through fluorescent boronic acid-based molecularly imprinted polymer. Anal. Chim. Acta 2017, 960, 110-116. [CrossRef] [PubMed] 
13. Terzopoulou, Z.; Papageorgiou, M.; Kyzas, G.Z.; Bikiaris, D.N.; Lambropoulou, D.A. Preparation of molecularly imprinted solid-phase microextraction fiber for the selective removal and extraction of the antiviral drug abacavir in environmental and biological matrices. Anal. Chim. Acta 2016, 913, 63-75. [CrossRef] [PubMed]

14. Speltini, A.; Scalabrini, A.; Maraschi, F.; Sturini, M.; Profumo, A. Newest applications of molecularly imprinted polymers for extraction of contaminants from environmental and food matrices: A review. Anal. Chim. Acta 2017, 974, 1-26. [CrossRef] [PubMed]

15. Rechichi, A.; Cristallini, C.; Vitale, U.; Ciardelli, G.; Barbani, N.; Vozzi, G.; Giusti, P. New biomedical devices with selective peptide recognition properties. Part 1: Characterization and cytotoxicity of molecularly imprinted polymers. J. Cell. Mol. Med. 2007, 11, 1367-1376. [CrossRef] [PubMed]

16. Morelli, I.; Chiono, V.; Vozzi, G.; Ciardelli, G.; Silvestri, D.; Giusti, P. Molecularly imprinted submicronspheres for applications in a novel model biosensor-film. Sens. Actuators B Chem. 2010, 150, 394-401. [CrossRef]

17. Habimana, J.D.D.; Ji, J.; Pi, F.; Karangwa, E.; Sun, J.; Guo, W.; Cui, F.; Shao, J.; Ntakirutimana, C.; Sun, X. A Class-specific Artificial Receptor-Based on Molecularly Imprinted Polymer-Coated Quantum Dot centers for the Detection of signaling molecules, N-Acyl-homoserine Lactones Present in Gram-Negative Bacteria. Anal. Chim. Acta 2018, 1031, 134-144. [CrossRef] [PubMed]

18. Yáñezsedeño, P.; Campuzano, S.; Pingarrón, J.M. Electrochemical sensors based on magnetic molecularly imprinted polymers: A review. Anal. Chim. Acta 2017, 960, 1-17. [CrossRef] [PubMed]

19. Wei, Y.B.; Tang, Q.; Gong, C.B.; Lam, M.H. Review of the recent progress in photoresponsive molecularly imprinted polymers containing azobenzene chromophores. Anal. Chim. Acta 2015, 900, 10-20. [CrossRef] [PubMed]

20. Yu, C.; Mosbach, K. Influence of mobile phase composition and cross-linking density on the enantiomeric recognition properties of molecularly imprinted polymers. J. Chromatogr. A 2000, 888, 63-72. [CrossRef]

21. Fang, G.Z.; Tan, J.; Yan, X.P. An ion-imprinted functionalized silica gel sorbent prepared by a surface imprinting technique combined with a sol-gel process for selective solid-phase extraction of cadmium(II). Anal. Chem. 2005, 77, 1734-1739. [CrossRef] [PubMed]

22. Graham, A.L.; Carlson, C.A.; Edmiston, P.L. Development and characterization of molecularly imprinted sol-gel materials for the selective detection of DDT. Anal. Chem. 2002, 74, 458-467. [CrossRef] [PubMed]

23. Wang, H.F.; Zhu, Y.Z.; Yan, X.P.; Gao, R.Y.; Zheng, J.Y. A Room Temperature Ionic Liquid (RTIL)-Mediated, Non-Hydrolytic Sol-Gel Methodology to Prepare Molecularly Imprinted, Silica-Based Hybrid Monoliths for Chiral Separation. Adv. Mater. 2006, 18, 3266-3270. [CrossRef]

24. Sun, X.; Lei, Y. Fluorescent carbon dots and their sensing applications. TrAC Trends Anal. Chem. 2017, 89, 163-180. [CrossRef]

25. 2Nd, P.T.; Zhang, Y.; Wang, T.H. Quantum dots in diagnostics and detection: Principles and paradigms. Analyst 2014, 139, 2968-2981.

26. Stanisavljevic, M.; Krizkova, S.; Vaculovicova, M.; Kizek, R.; Adam, V. Quantum dots-fluorescence resonance energy transfer-based nanosensors and their application. Biosens. Bioelectron. 2015, 74, 562-574. [CrossRef] [PubMed]

27. Foubert, A.; Beloglazova, N.V.; Saeger, S.D. Comparative study of colloidal gold and quantum dots as labels for multiplex screening tests for multi-mycotoxin detection. Anal. Chim. Acta 2016, 955, 48-57. [CrossRef] [PubMed]

28. Himaja, A.L.; Karthik, P.S.; Singh, S.P. Carbon Dots: The Newest Member of the Carbon Nanomaterials Family. Chem. Rec. 2015, 15, 595-615. [CrossRef] [PubMed]

29. Mosconi, D.; Mazzier, D.; Silvestrini, S.; Privitera, A.; Marega, C.; Franco, L.; Moretto, A. Synthesis and Photochemical Applications of Processable Polymers Enclosing Photoluminescent Carbon Quantum Dots. ACS Nano 2015, 9, 4156-4164. [CrossRef] [PubMed]

30. Wang, F.; Pang, S.; Wang, L.; Li, Q.; Kreiter, M.; Liu, C. One-Step Synthesis of Highly Luminescent Carbon Dots in Noncoordinating Solvents. Chem. Mater. 2010, 22, 4528-4530. [CrossRef]

31. Zhou, Y.; Qu, Z.B.; Zeng, Y.; Zhou, T.; Shi, G. A novel composite of graphene quantum dots and molecularly imprinted polymer for fluorescent detection of paranitrophenol. Biosens. Bioelectron. 2014, 52, 317-323. [CrossRef] [PubMed] 
32. Zor, E.; Morales-Narvaez, E.; Zamora-Galvez, A.; Bingol, H.; Ersoz, M.; Merkoci, A. Graphene Quantum Dots-based Photoluminescent Sensor: A Multifunctional Composite for Pesticide Detection. ACS Appl. Mater. Interfaces 2015, 7, 20272-20279. [CrossRef] [PubMed]

33. Cayuela, A.; Soriano, M.L.; Carrillo-Carrion, C.; Valcarcel, M. Semiconductor and carbon-based fluorescent nanodots: The need for consistency. Chem. Commun. 2016, 52, 1311-1326. [CrossRef] [PubMed]

34. Xue, M.; Zou, M.; Zhao, J.; Zhan, Z.; Zhao, S. Green preparation of fluorescent carbon dots from lychee seeds and their application for the selective detection of methylene blue and imaging in living cells. J. Mater. Chem. B 2015, 3, 6783-6789. [CrossRef]

35. Zhao, A.; Chen, Z.; Zhao, C.; Gao, N.; Ren, J.; Qu, X. Recent advances in bioapplications of C-dots. Carbon 2015, 85, 309-327. [CrossRef]

36. Wang, F.; Xie, Z.; Zhang, H.; Liu, C.Y.; Zhang, Y.G. Highly Luminescent Organosilane-Functionalized Carbon Dots. Adv. Funct. Mater. 2011, 21, 1027-1031. [CrossRef]

37. Sun, J.; Zhuang, J.; Guan, S.; Yang, W. Synthesis of robust water-soluble ZnS:Mn/SiO 2 core/shell nanoparticles. J. Nanopart. Res. 2008, 10, 653-658. [CrossRef]

38. Wang, X.; Cao, L.; Lu, F.; Meziani, M.J.; Li, H.; Qi, G.; Zhou, B.; Harruff, B.A.; Kermarrec, F.; Sun, Y.P. Photoinduced electron transfers with carbon dots. Chem. Commun. 2009, 46, 3774-3776. [CrossRef] [PubMed]

39. Wang, H.F.; He, Y.; Ji, T.R.; Yan, X.P. Surface molecular imprinting on Mn-doped ZnS quantum dots for room-temperature phosphorescence optosensing of pentachlorophenol in water. Anal. Chem. 2009, 81, 1615-1621. [CrossRef] [PubMed]

40. Xu, S.; Lu, H. Mesoporous structured MIPs@CDs fluorescence sensor for highly sensitive detection of TNT. Biosens. Bioelectron. 2016, 85, 950-956. [CrossRef] [PubMed]

41. Li, L.; Ren, S.; Shao, M.; De Saeger, S.; Song, S.; Yan, L. A competitive immunoassay for zearalenone with integrated poly(dimethysiloxane) based microarray assay. Anal. Methods 2018, 10, 4036-4043. [CrossRef]

(C) 2018 by the authors. Licensee MDPI, Basel, Switzerland. This article is an open access article distributed under the terms and conditions of the Creative Commons Attribution (CC BY) license (http:/ / creativecommons.org/licenses/by/4.0/). 